\title{
Augmented Cognition via Brainwave Entrainment in Virtual Reality: An Open, Integrated Brain Augmentation in a Neuroscience System Approach
}

\author{
Emanuele Argento $^{1}$ - George Papagiannakis ${ }^{1,2} \cdot$ Eva Baka $^{1,3} \cdot$ Michail Maniadakis $^{1}$. \\ Panos Trahanias $^{1,2} \cdot$ Michael Sfakianakis $^{4} \cdot$ Ioannis Nestoros $^{5}$
}

Received: 6 September 2016/Accepted: 15 February 2017/Published online: 28 February 2017

(C) Springer SBM Singapore Private Ltd. 2017

\begin{abstract}
Building on augmented cognition theory and technology, our novel contribution in this work enables accelerated, certain brain functions related to task performance as well as their enhancement. We integrated in an open-source framework, latest immersive virtual reality (VR) head-mounted displays, with the Emotiv EPOC EEG headset in an open neuro- and biofeedback system for cognitive state detection and augmentation. Our novel methodology allows to significantly accelerate content presentation in immersive VR, while lowering brain frequency at alpha level-without losing content retention by the user. In our pilot experiments, we tested our innovative VR platform by presenting to $N=25$ subjects a complex 3D maze test and different learning procedures for them on how to exit it. The subjects exposed to our VR-induced entrainment learning technology performed significantly better than those exposed to other "classical" learning procedures. In particular, cognitive task performance augmentation was measured for: learning time, complex navigational skills and decision-making abilities, orientation ability.
\end{abstract}

Eva Baka

eva.mpaka@gmail.com

1 Computational Vision and Robotics Laboratory, Institute of Computer Science, Foundation for Research and Technology, Heraklion, Greece

2 Department of Computer Science, University of Crete, Heraklion, Greece

3 Department of Medicine, University of Greece, Heraklion, Greece

4 Department of Business Administration, University of Piraeus, Piraeus, Greece

5 Synchronal Amphiaraia, University of Crete Snip-off Company, Athens, Greece
Keywords Augmented cognition - Brainwave entrainment · Virtual reality platform - Technologyenhanced learning · Human-computer interaction · Brain-computer interfaces

\section{Introduction}

The focus of augmented cognition systems is real-time assisted human interaction with complex computer systems based on biofeedback detection and manipulation [40]. The efficacy of this scientific approach to human abilities enhancement has been widely proved over more than a decade of independent research at DARPA [34, 35, 41].

Even if we recognize the importance and utility of such an approach in augmenting human cognition, there is strong evidence as suggested in this paper that recent VRenabled technologies can enhance human performance permanently and generally and not only-as augmented cognition does-temporarily and in synergy with the specific technology used at the moment of the humancomputer interaction. Neuro- and biofeedback training systems also have been widely investigated, tested and used as an effective method for cognitive, creative and physical human enhancement in performance oriented contexts $[15,16,33,42]$ but not extensively combined with latest VR developments [8].

Our approach, while building upon the already rich research on systems neuroscience, brain augmentation, augmented cognition and Neurofeedback training, bridges them for the first time into a novel integrated technology via new interdisciplinary research that effectively combines neuroscience, computer science, engineering, psychology and education, in order to produce scientific theories, methodologies and technological tools, aiming to 
enhance human skills, learning potential and intelligent problem-solving abilities.

This paper is structured as following: "Materials and Methods" section briefly describes our novel methodology in the frame of augmented cognition concepts, while "Results of the Experiment" section explains in essence why our approach is interesting and scientifically relevant. "Discussion" section describes our system with more details as well as it describes our experiment and findings. At the end, we draw some final conclusions and propose future developments. The statistical significance of the research might be arguable, as the experimental group was limited to only 25 subjects, the experimental groups many (5) and the experimental conditions complex (a complex VR environment with many variables). Nevertheless, our main objective is the practical realization of a "proof of concept" which is by definition usually focused. The purpose of this preliminary prototype and research is to prove a concept that the theoretical idea of fast learning and skills enhancement in VR is-under the described certain conditions-feasible and has practical huge potential.

\section{Materials and Methods}

Our novel methodology uses an open virtual reality immersive platform (VR) as an auditory-visual interface. Through manipulation of virtual space and time in VR, we embarked on the ambitious research (with first promising results) on how we can visually and auditory "download" learning content to the brain, interactively, by varying in VR the speed and spatial modality of content presentation. Such speed and modality vary accordingly to a complex set of neuro- and biofeedbacks, interactively detected, modified and optimized by our real-time system for Autonomous Cognitive State Detection, Manipulation and Augmentation.

By applying our novel VR technology, we illustrate first findings on how to significantly compress the time needed to develop certain ability or absorb a certain knowledge (neuroadaptive accelerated learning), while at the same time permanently improving the performance for such skill or area of knowledge (permanent enhancement of human cognition).

Hence, we aim to establish a novel research area of augmented cognitive neuro- and biofeedback, where adaptive, fast learning VR systems can be employed for permanent and independent human cognitive enhancement.

\section{The Context and Need of Application of Our Novel Technology}

Exponentially growing advances in computational platforms advancement create the fastest changing environment ever in history, influencing every single aspect of human life. The main technological advancements in our Information Age that most require fast human adaptation response are, among the others:

- The technological singularity and the fast rise of strong artificial intelligence that might potentially create the need of augmented cognition human abilities of control over AI [44].

- Big data exponential development that might potentially create the necessity of autonomous human fast decision skills in a complex digital interconnected environment [19].

- Fast adaptation and accelerated learning might become increasingly intertwined and both essential in our modern technological society [4].

We do not simply need to adapt or learn something once and for all, (as this "something" is always changing...). In order to adapt to the Information Age, we need to rethink the process of how to learn better and faster, or-in other word-we need to become superfast learners, and apply this "advanced learning technology" to a wide variety of skills and problem-solving contexts.

Old and classical ways of learning (created before the modern information Age) are no longer efficient in terms of volume/quantity of transmitted knowledge and speed of absorption we need nowadays [2].

Therefore, we developed a novel architecture for augmented cognition by integrating latest virtual reality (VR) immersive head-mounted displays with mobile neuro- and biofeedback systems for Real-time, Autonomous Cognitive State Detection, Manipulation and Augmentation. We also integrated in our open-source VR framework both experimental Oculus Rift HMDs and the Emotiv EPOC mobile EEG headset (Fig. 1).

More specifically our novel VR platform for augmented cognition consists of:

- A noninvasive neuro- and biofeedback systems for Real-time, Autonomous Cognitive State Detection and Manipulation;

- Virtual immersive reality for content delivery, cognitive enhancement, active interaction and cognitive state manipulation;

- Open-source VR framework for real-time 3D content delivery, fast and effective learning and human cognitive enhancement for memory, knowledge and skills;

- A neuro- and biofeedback system that can regulate in real-time content delivery and learning engines according to real-time cognitive state detection.

One of the most important ideas of our research is the possibility of delivering at high-speed audiovisual content for learning and cognitive enhancement purposes, without 
Fig. 1 Overview of our novel, open VR platform (top) with the associated wearable $\mathrm{h} / \mathrm{w}$ devices (Oculus Rift HMD and Emotiv EEG headset) worn by a subject of our experiments (center, bottom). A detailed description of the class diagram of the VR architecture is provided in $[23,29,30]$
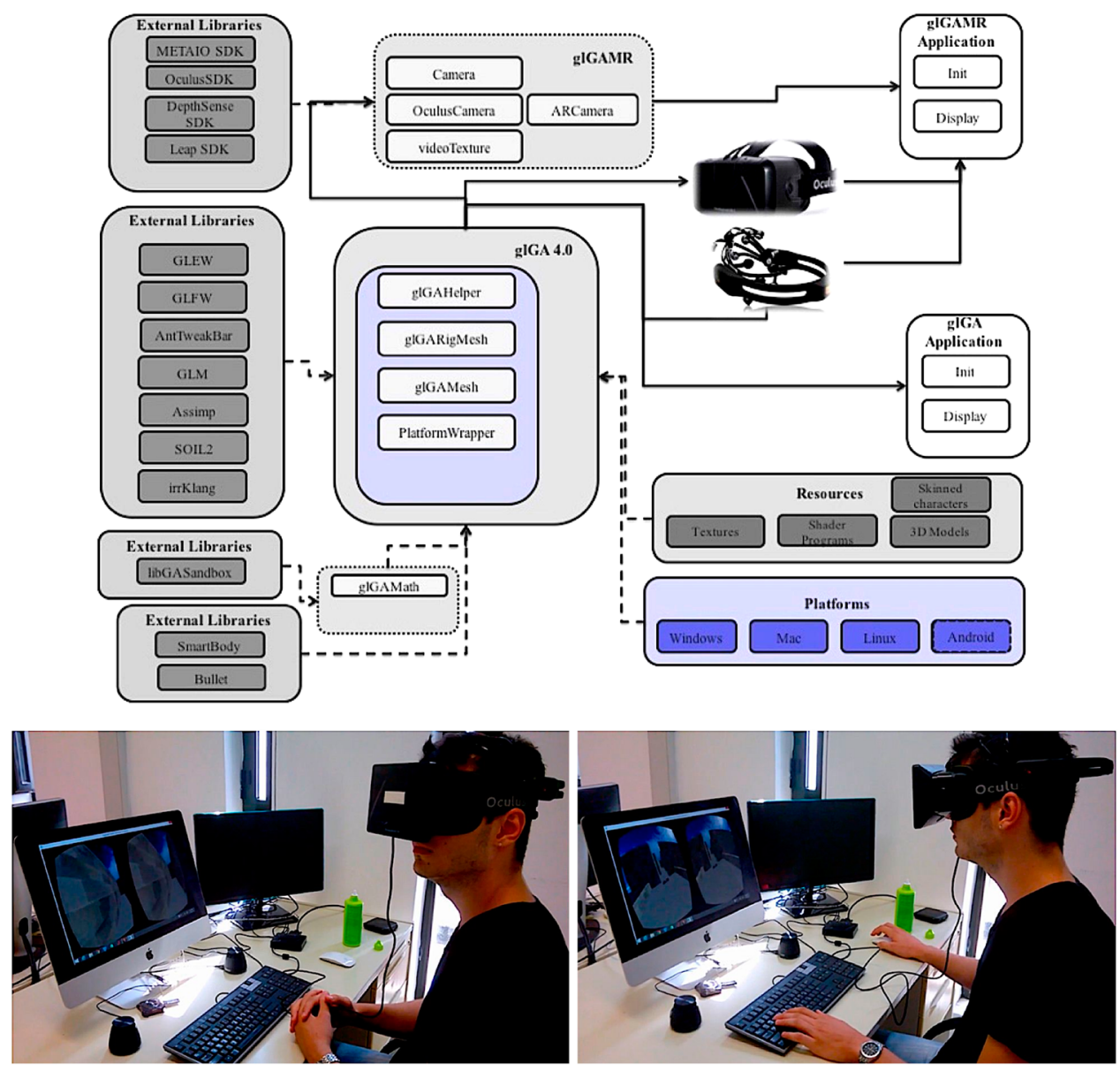

losing knowledge retention from the subject. In fact, while a subject is immersed in VR, we can control the speed at which content is presented, the same way we can manipulate the speed when we see a movie on a computer screen. Our pilot experiment has shown that this strategy, when applied in virtual reality and combined with cognitive state detection and manipulation via audio rhythmic brain stimulation, is highly effective in both reducing learning time and improving performance at the same time. The reason we think this is possible, and effective is inherent with the concept itself of virtual reality.

Since content presented in a high realistic VR environment is the actual reality for the subject, accelerating content presentation in VR is equivalent to "accelerating speed of events in (virtual) reality." By rephrasing Einsteinian relativistic concepts, we might say that the higher the speed of events in VR, the slower the time flows in VR, compared to the reality outside VR. By interconnecting VR with a neuro- and biofeedback engine, we can create a neuroadaptive content presentation technology that can optimize and enhance the human ability of learning in terms of time.

As time and space perception is actually a qualia, or a subjective perception [11], we believe that by slowing brainwave toward the alpha frequency-even if subjective time perception in VR might not change in comparison with normal reality time perception-events at higher speed in VR can be effectively processed by the subject's brain for learning and cognitive enhancement purposes, therefore making such processes faster.

Our pilot experiment does not only show that this is possible, but also points out that accelerating events in VR while slowing brainwaves toward the alpha frequency creates a compound effect that makes the entrainment fast learning process as effective as the normal learning (but less time-consuming) and even much more effective for direct and indirect cognitive and skill enhancement.

Slowing brainwaves toward the alpha frequency $[38,39]$ can be done in different ways, ranging from invasive chemical compounds, to transcranial electrical brain stimulation, to noninvasive audiovisual rhythmic stimulation that can theoretically produce a phenomenon called: "brainwave entrainment." Brainwave entrainment can be achieved, in general, through photic or audio stimulation and has an old story. In fact, the first known clinical application belongs to the French psychologist Pierre Janet, at the turn of the twentieth century, in the late $1800 \mathrm{~s}$. A lot of studies followed afterward by many well respected 
researchers such as Bartley [14], Goldman et al. [9], Jung [17], Toman [33]. However, no one ever considered the possible effects of a photic stimulation. Therefore, after the initial demonstration of Berger [13] in 1934 on electrical activity recorded from the human brain, Adrian and Mathews [1] showed that the Berger rhythm, which means alpha rhythm, could be further amplified by photic stimulation at the same frequency. In 1942, Dempsey and Morison [7] found that brain entrainment could also be induced by tactile stimulus and in 1959 Chaitran [7] reported entrainment effects with an auditory stimulus. Meanwhile, in 1956, Walter [43] published the results of his researches on the emotional and phychological effects of a flickering light stimulation. Flickering light produced frequency-dependent sensations of pattern, movement and color. The development of brain entrainment tools increased greatly from 1973, with Oster's result [28] which evidenced the properties of binaural beats. Studies on the effects of brain entrainment on situations like pain, headaches, migraines, anxiety and stress followed in the 1980s, as well as the incorporation of specific devices which directly allowed the achievement of an entrainment, such as David Siever's device [12]. This kind of researches expanded in the 1990s to include learning and memory, learning disabilities, attention deficit hyperactivity disorder, behavioral problems and PMS [6, 11, 12, 21, 37].

At the moment, while investigating other options, for our novel technology, we have chosen this method as it is easy to implement and because there is enough scientific research on the subject that proves its effectiveness [20]. The stimulus used was a simple isochronic tone, recorded at a specific target frequency of $10 \mathrm{~Hz}$ on a mp3 file and played into the ears of the subjects with normal/commercial earphones.

\section{The Experiment}

While the primary objective of our technology is human cognition augmentation and improvement in task performance, we first employed our novel architecture to a common orientation path-finding task and way-finding problem-solving abilities in immersive VR with and without audio rhythmic brain stimulation.

Our simple pilot experiment, used to test our idea, is presented in this section.

\section{General Overview and Description of the Experiment}

The main idea of the experiment was to test the ability of a subject to effectively learn how to get out of a maze in an immersive VR environment.
Different learning processes were implemented and tested, in order to determine the most effective one for learning and augmented cognition. The effectiveness of each learning process was measured by the time needed by the subject to reach a specific point (a landmark) in the labyrinth, while trying to find its exit. Time to exit was also measured and was used to evaluate the ability to perform precise movements in the labyrinth and effectively recovering from accidental mistakes as indirect by-product of the learning process.

$N=25$ subjects ( 16 males and 9 females-age from 13 to 52) were equally divided into 5 experimental groups of 5 subjects each:

- Control group (C-group);

- Basic pre-task entrainment group (B-group);

- Simple learning group (SL-group);

- Entrainment learning group (E-group);

- Entrainment fast learning group (EFL-group).

The mean age of each group was in the range 26-35.

Because 3D gaming experience and proficiency of each subject could play an important role in the average performance of the group, all the participants were first asked to self-evaluate their proficiency/experience in 3D gaming on a scale from 0 -no experience at all-to 5-maximum possible experience level. Based on their self-evaluation, the participants were then randomly assigned to one of the 5 experimental groups, in order to maintain the mean proficiency/experience level of every group at a level of 2-2.5.

The experiment was a single blind one, where the individual subjects did not know whether they were "test" subjects or members of an "experimental control" group. All the participants were informed that their task was to find the exit root from a labyrinth, presented to them in a VR environment via Oculus Rift, in the shortest time possible. All groups were given the exact same labyrinth. In order to minimize anxiety during their performance, all the participants were informed that their performance would not be used to evaluate their intelligence or abilities in any way but that the purpose of the experiment was simply to measure EEG in different VR environments and gaming situations.

Every subject participating to the experiment was first presented with Tuscany, a well-known VR environment in Oculus-in which he/she was asked to move around for about $5 \mathrm{~min}$, just to familiarize with 3D virtual reality in Oculus and the navigation buttons. Navigation in VR, both for "Tuscany" and the maze, was performed by using the mouse for rotation and the keys (w-s-a-d) to move, respectively, forward, backward, left and right, as in usual 3D video games. At the present stage of development, this choice of navigation is the simplest possible in an 
experimental VR environment with Oculus Rift. Of course, people accustomed to video games find this way (or any other possible way of navigation) easier to use than people with less experience and that is why each experimental group has been balanced. Even though different subjects might have different 3D-game experience, we managed to have a roughly same average $3 \mathrm{D}$ game experience level of each group. Moreover, Tuscany is an open environment (a minimalist villa in a relaxing open garden). Due to such openness, navigation inside Tuscany is quite easy. On the contrary, a maze is a closed environment, with narrow walls hindering freedom of movements; to make navigation in the labyrinth even more difficult, in the second half of it (see why in the description of the labyrinth), by design collision detection for the walls was not accurate. By choosing such open environment as "familiarization" for all the experimental subjects, we avoided mixing the familiarization procedure with actual training for the labyrinth. This helped in establishing a better baseline for the entire experiment.

The maze was built in order to present a moderate level of difficulty to reach the exit. Two landmarks were put in the labyrinth: one at one-third of the maze, one to mark the exit. The first part of the maze-till the first landmark-was actually the part involving decisions on the correct route to take. The second part of the maze — after the first landmarkwas actually straightforward, with just one single possible path to follow till the exit of the labyrinth. By design, collision detection of the walls throughout the entire second part of the maze was incorrect: In fact on some points, the walls were either sticky or it was possible passing through them. This created for the participants serious navigational challenges that had nothing do to with the previous learning process in itself, but with the ability to perform precise movements in the labyrinth and effectively recovering from accidental mistakes. We decided to have "anomalous" collision detection issues after the path planning part of the maze especially and purposely to experimentally reverse a classical situation where the latter is usually considered when the first one is "solved." In fact the collision problems of the first part of the maze are those normally found in a "normal" 3D game, where the player is simply trying to avoid hitting walls and find his/her way out of the maze. On the contrary, the collision problems of the second part of the maze are "anomalous" due to the purposely creation and implementation of walls that are semi-permeable and "sticky" (not as real wall would be). When the player by mistake "hits" those semi-permeable and "sticky" walls, he/ she is partially "captured" inside the wall: The player has to perform exact precise movements to get free of the wall and continue on his/her path to the exit. Because of this especially difficult wall structure (only possible to create in a virtual 3D environment and not in a real world where wall are not permeable nor sticky), and because the second part of the maze is just one-way to the exit, the only parameter actually measured by the time needed to get to the exit in the second part of the maze is the ability to accurately navigate and effectively recover from mistakes.

Therefore, the effectiveness of the different learning processes (see following description of the experimental groups) could only be evaluated by the time needed to reach the first landmark, while final exit time (or better the delta between final exit time and time to first landmark) could be used to evaluate ability to perform precise movements in the labyrinth and effectively recovering from accidental mistakes.

The learning process was basically having all the subjects watching passively a first-person camera flying through the labyrinth from the entrance directly to the exit, through the only possible path. We had 2 possible speed/duration times for a single fly-through: the normal speed where a single fly-through would take approximately $1 \mathrm{~min}$ and $45 \mathrm{~s}$ and the fast speed where a single flythrough would take approximately $50 \mathrm{~s}$.

During the learning session, we presented each subject with 3 fly-throughs. The SL-group and EL-group were presented with fly-throughs at normal speed. The EFLgroup was presented with fly-throughs at fast speed. The B-, EL- and EFL-groups were also administrated with rhythmic brain stimulation before the learning session.

Audio rhythmic stimulation was performed by presenting the subjects via headphones with a continuous audio impulse at a frequency of $10 \mathrm{~Hz}$. At the same time, a relaxing environment in VR was presented in Oculus VR. The relaxing VR environment was the same for all the participants undergoing this procedure.

After that, and only for the subjects in the EL- and EFL-groups, the rhythmic brain stimulation was again presented at the same frequency of $10 \mathrm{~Hz}$ via headphones, while this time undergoes the learning phase. The C-and SL-groups were not presented with any audio rhythmic stimulation.

The C-group after the familiarization procedure was simply administrated the labyrinth test. The time needed for each of the experimental subject in this group to find the halfway mark and exit the maze was then recorded; then, the mean value of the group calculated to determine a base line for our experiment.

The B-group after the familiarization procedure was first administrated with 15 min of audiovisual rhythmic brain stimulation in the relaxing VR environment mentioned before, at $10 \mathrm{~Hz}$ frequency. Then, the test was administrated. The time needed of the participants to find the halfway mark and exit the maze was then recorded; then, the mean value of the group was calculated to measure the effectiveness of rhythmic brain stimulation alone on the labyrinth task (Table 1). 
Table 1 Characteristics for each group

\begin{tabular}{|c|c|c|c|c|c|}
\hline \multirow[t]{2}{*}{ Group name } & \multicolumn{5}{|l|}{ Task } \\
\hline & Familiarization & Relaxation & Learning & Fast learning & Maze \\
\hline $\mathrm{C}$ & $\checkmark$ & & & & $\checkmark$ \\
\hline B & $\checkmark$ & $\checkmark$ & & & $\checkmark$ \\
\hline SL & $\checkmark$ & & $\checkmark$ & & $\checkmark$ \\
\hline EL & $\boldsymbol{V}$ & $\checkmark$ & $\nu$ & & $\checkmark$ \\
\hline EFL & $\boldsymbol{V}$ & $\boldsymbol{V}$ & & $\boldsymbol{V}$ & $\checkmark$ \\
\hline
\end{tabular}

The SL-group-or the "second control group"-after the familiarization procedure watched a learning video at normal speed, presented 3 times. The labyrinth test was then administrated to the SL-subjects. The time needed for each of them to find the halfway mark and exit the maze was then recorded as well, and the mean value was used to evaluate the effectiveness of a simple learning process (at normal speed and without rhythmic brain stimulation) in order to have a second base life by confronting this classical learning procedure with the EL and EFL procedures.

The EL-group after the familiarization procedure was first administrated with 15 min of audio rhythmic brain stimulation in the relaxing VR environment at $10 \mathrm{~Hz}$ frequency, followed by a learning video at normal speed presented 3 times with embedded audio rhythmic brain stimulation. Finally, the labyrinth test was administrated to the EL-group and the time needed for each of the experimental subject in this group to find the halfway mark and exit the maze was then recorded. The mean calculated value of all the subjects of the group was used to evaluate the effectiveness of rhythmic brain stimulation and learning at normal speed in the labyrinth task.

The EFL-group, like the EL-group, was first administrated with 15 min of audio rhythmic brain stimulation in the relaxing VR environment at $10 \mathrm{~Hz}$ frequency. After that, a learning video at fast speed was presented 3 times with embedded audio rhythmic brain stimulation. Finally, the labyrinth test was administrated to the EFL-group. The time needed for each of the experimental subject in this group to find the halfway mark and exit the maze was then recorded, so that the mean value was calculated to evaluate the effectiveness of rhythmic brain stimulation and timecompressed learning (fast speed) on the labyrinth task.

For all the groups, if time spent in the labyrinth (without finding the exit or the halfway landmark) was more than 6 min then the experiment was interrupted and no exit or halfway landmark time was recorded (see sections: "Statistics" and "Results" for more details on how those special cases where treated).

\section{Signal Processing Procedure}

After having recorded the EEG data, EEGlab and NBT were used for signal processing and data analysis. EEGlab, as well as NBT, is a toolbox and graphic user interface, running on top of the MATLAB environment, developed for processing collections of single-trial and/or averaged EEG data of any number of channels [9, 10].

To filter the data as well as clean them from any possible artifacts, the preprocessing tools were employed, offered by both EEGlab and NBT [9] since sampling of the data lead to aliasing. In general, for being able to eliminate this aliasing process, it is suggested in the bibliography that the data should be low-pass filtered at frequencies at least onethird lower than the sampling rate $[3,7]$. Given that in our case the sampling rate was $128 \mathrm{~Hz}$, we used a basic FIR filter with the lower edge of the frequency at $45 \mathrm{~Hz}$ and the higher one at $55 \mathrm{~Hz}$.

To clean the data, we removed further artifacts induced by eye blinking and abrupt head or body movements throughout the entire process. The Emotiv headset allowed every participant to move freely in every direction, but this caused most of the times unavoidable artifacts. Given the purpose of our study, those movements could not be eliminated at their entirety. For an efficient and a more promising cleaning, we, sometimes had also to reject a number of erroneous channels, marked as such by the toolkits.

In addition, we run ICA for data decomposition, which helped us with the further EEG processing. In this case, EEGlab offered the most reliable results composed of 14 components, as the exact number of the channels used. Subsequently, examining components activation and their maps, we removed the erroneous components.

Through this procedure, we were able to determine the frequencies that corresponded to the microvolts we recorded with the Emotiv headset throughout the experiment. At the end, it allowed us to plot either the spectrum of each channel or the one of the whole signal and the activity of each channel in relation to the proportional brain activity area.

We used the Emotiv headset because it is a low-cost and high-quality EEG technology. Researchers do not all agree on the reliability of the Emotiv EPOC headset for complex tasks like-for example_rehabilitation or prosthesis control, but for simple noncritical applications such as games and communication systems, "this low-cost headset is able 
to record EEG data in a satisfying manner" [12]. As previously explained in this section, we relied on postrecording accurate EEG data processing to compensate for possible real-time inaccurate readings of the Emotiv headset.

\section{Statistics}

Data are expressed as mean values $\pm 1 \mathrm{SD}$. Analysis of variance combined with multiple range analysis according to Fisher's least significant difference (LSD) procedure or Kruskal-Wallis test combined with Box-and-Whisker Plot (median notch option) was used as appropriate. A $p$ value of less than 0.05 was considered statistically significant.

\section{Results of the Experiment}

The following table summarizes the experimental results in terms of performance in the labyrinth task and learning time.

Analysis and discussion of those results are presented in the next paragraph.

In Table 2, "n.a." (not applicable) means that the time needed to exit the labyrinth exceeded the time limit of $6 \mathrm{~min}$ and was considered as "missing variables" in the statistical analysis. Thus, the EL-group in the TOT variable (total time to exit) participates with only 3 values and the B group with only 1 value and hence we do not allocate to $\mathrm{B}$ group the mean values or participate in the ANOVA analysis regarding the TOT variable but only the T1 (average time to halfway mark) (Fig. 2).

- Meaning of the Delta (difference between the halfway mark time and the exit time in seconds)

We remind the reader that-by design-from the halfway mark to the exit there are no choices to make, it is simply one-way to the end. We remind the reader also that the learning phase has only to do with showing, to a passive subject, the path to the exit and has nothing to do with actual practice in the labyrinth (see also consideration about the familiarization phase). So we can consider the difference between the halfway mark time and the exit time (the delta) as a possible measure of the ability of a subject to perform precise movements in the labyrinth and effectively recovering from accidental mistakes (precision and recovery ability).

Concerning EEG analysis for C-group, the obtained results were within a range of frequencies from about 11 to $17 \mathrm{~Hz}$. There were no big differences between the familiarization and maze tasks, which means that participants almost maintained their primary frequency throughout the procedure. If, for example, the power spectrum of a participant presented peak at about $17 \mathrm{~Hz}$ in the familiarization task, then, in the maze task, the frequency will peak at almost $16 \mathrm{~Hz}$. We noticed though that at certain occasions, in the familiarization task, there were multiple peaks for the same spectrum, but usually concerning the same brainwave state, which in our case was alpha or lower beta (Fig. 3).

In the basic entrainment group (B), we found similar results concerning the familiarization task. The power spectrum presented an average peak at $15 \mathrm{~Hz}$. After having applied brainwave entrainment through the use of the isochronic beat mentioned before, we noticed changes in the associated EEG data. The spectrum peaked at $11-12 \mathrm{~Hz}$, and we were able to verify this result through the spectrum plot given by EEGlab and NBT and through the activity of each channel individually.

In Fig. 4, we are able to see the power spectrum of an individual channel and, also, its activity. The colors on top present this activity based on each color, as we mentioned before for the scalp map. The red one witnesses the highest possible activity of the specific brain area in the indicated frequency. Near this box, there is a scale for deciphering the mean of each color. The graph below presents the

Table 2 Results per experiment group

\begin{tabular}{|c|c|c|c|c|c|}
\hline $\begin{array}{l}\text { Experimental group } \\
\text { ID }\end{array}$ & $\begin{array}{l}\text { Average 3D gaming } \\
\text { experience }\end{array}$ & $\begin{array}{l}\text { Average } \\
\text { age }\end{array}$ & Average time to halfway mark (T1) & $\begin{array}{l}\text { Average time to exit } \\
\text { (TOT) }\end{array}$ & Delta \\
\hline $\mathrm{C}$ & 2.25 & 28.25 & $\begin{array}{l}35.50 \mathrm{~s} \\
\sigma=4.80\end{array}$ & $\begin{array}{l}226.75 \mathrm{~s} \\
\sigma=4.72\end{array}$ & $\begin{array}{l}191.25 \mathrm{~s} \\
\sigma=3.72\end{array}$ \\
\hline B & 2.25 & 32.75 & $\begin{array}{l}168.00 \mathrm{~s} \\
\sigma=4.65\end{array}$ & $\begin{array}{l}\text { n.a. } \\
>6 \text { min }\end{array}$ & n.a. \\
\hline SL & 2.375 & 34.75 & $\begin{array}{l}25.25 \mathrm{~s} \\
\sigma=1.71\end{array}$ & $\begin{array}{l}102 \mathrm{~s} \\
\sigma=1.83\end{array}$ & $\begin{array}{l}76.75 \mathrm{~s} \\
\sigma=1.82\end{array}$ \\
\hline EL & 2.125 & 29.5 & $\begin{array}{l}23.50 \mathrm{~s} \\
\sigma=2.38\end{array}$ & $\begin{array}{l}69.6 \mathrm{~s} \\
\sigma=2.52\end{array}$ & $\begin{array}{l}46.10 \\
\sigma=2.12\end{array}$ \\
\hline EFL & 2.375 & 26.5 & $\begin{array}{l}15.25 \mathrm{~s} \\
\sigma=1.50\end{array}$ & $\begin{array}{l}74.75 \mathrm{~s} \\
\sigma=2.06\end{array}$ & $\begin{array}{l}59.50 \\
\sigma=1.89\end{array}$ \\
\hline
\end{tabular}


Fig. 2 Histogram showing the differences of $\mathrm{T} 1$ time according to each group

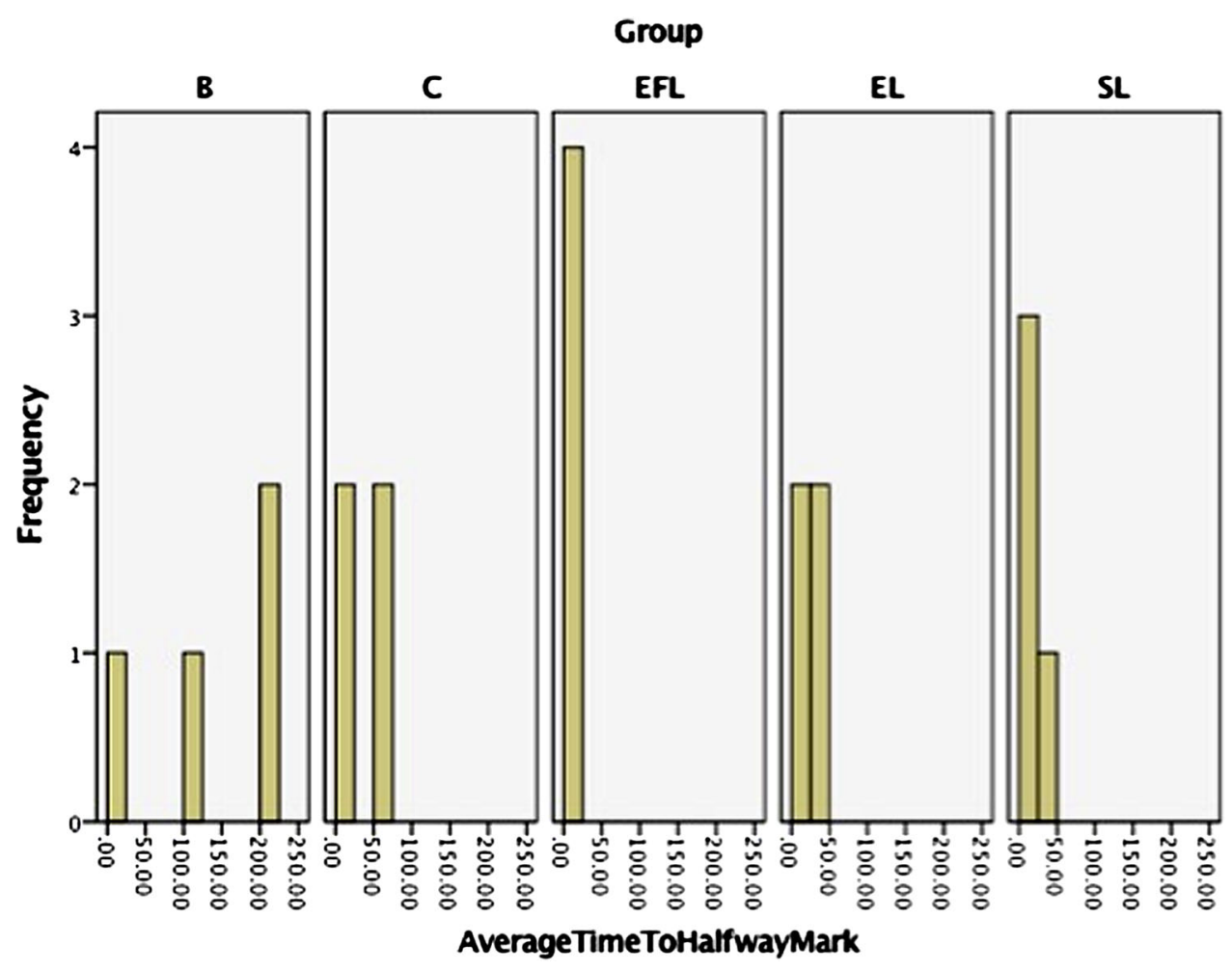

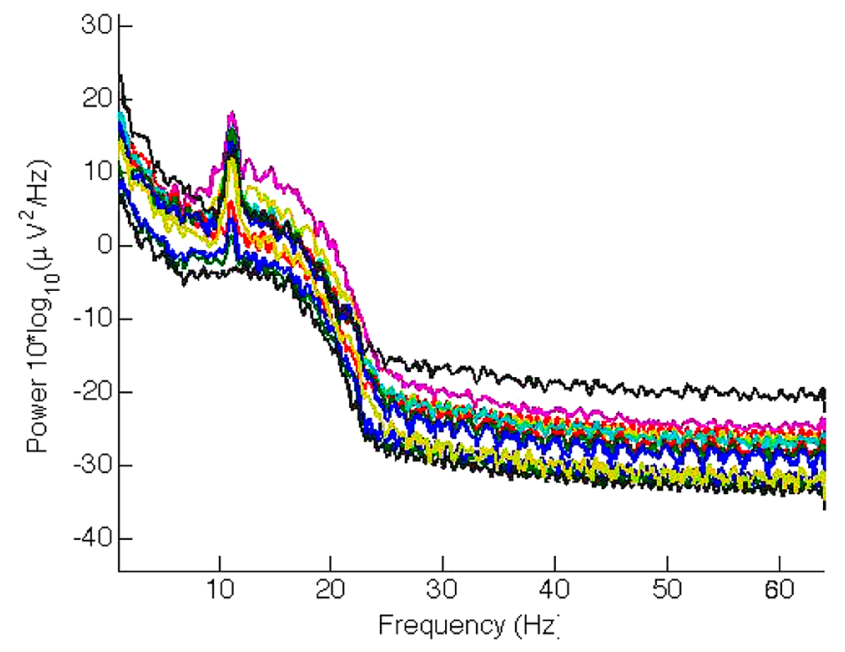

Fig. 3 Power spectrum for familiarization task in C-group using EEGLab. The several colored lines show the respective channels. The scalp map indicates the activity of the specific frequency in different brain areas. The red color represents the maximum intense activity (C-group-familiarization task)

power spectrum of the specific channel, and we are clearly able to see that, in this example, frequency peaks at $12 \mathrm{~Hz}$. Finally, the dots in the right present the position of each channel on the scalp.

In the familiarization task of SL-group, we observed almost the same results, with an average spectrum peak at $12 \mathrm{~Hz}$. In this group, there was no relaxation task, so the procedure proceeds with the simply learning task. All the participants, throughout this task, presented a peak at $11.5 \mathrm{~Hz}$, which is clearly an alpha state (Fig. 5).

In the relaxation task, the participants of EL-group managed to find themselves slightly in a lower range of frequency peaking at $10 \mathrm{~Hz}$. During the next task of learning, the peak of the frequency was almost maintained at $10-11 \mathrm{~Hz}$ (Fig. 6).

Figures 7, 8, 9 present a complete image of the relaxation task of EFL-group, using both EEGlab and NBT. It is clearly indicated that the frequency has been maintained at $10 \mathrm{~Hz}$, as it was in the previous task of familiarization. During the next tasks, we observed a range of frequencies from 8 to $12 \mathrm{~Hz}$.

\section{Analysis of the Experimental Results}

Various studies and research experiments have been conducted on the effects of audiovisual rhythmic stimulation at alpha frequency on EEG and performance [14, 15, 17, 45].

Some studies also have also shown changes in brainwaves toward the alpha frequency after exposure to television [24] and video games [32], and others have proved that subjects showing alpha frequency brainwave dominance learn faster while playing a video game developed for cognitive research [26].

Lastly, a series of studies have demonstrated that Zen meditation (Zazen) [22] and nondirective meditation [25] 


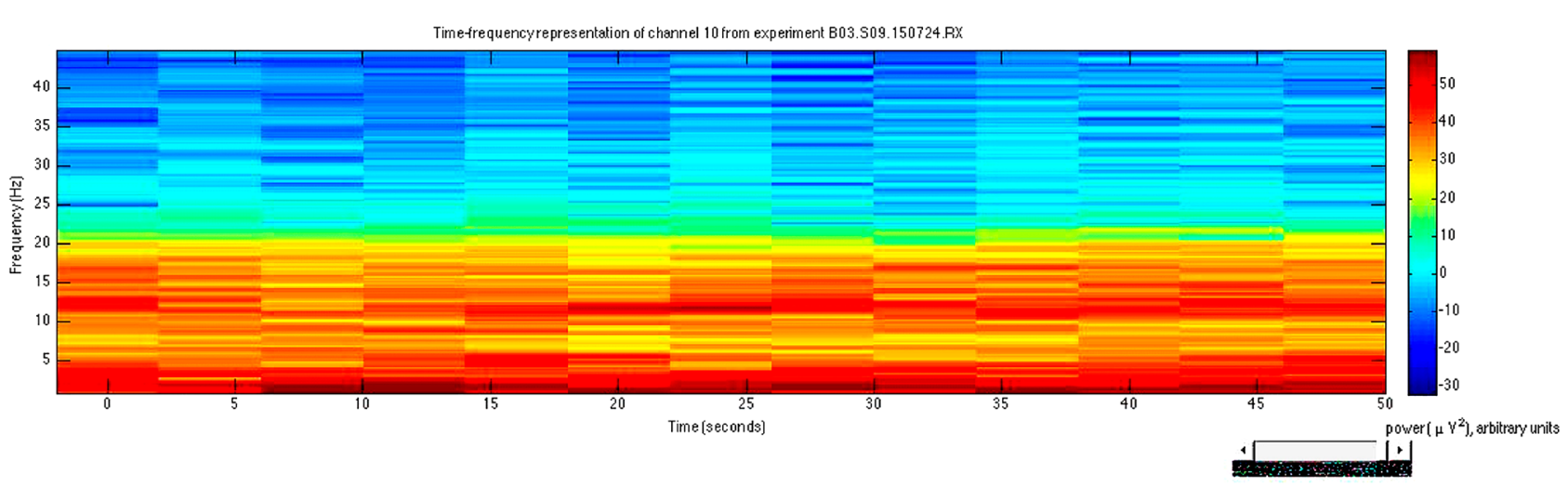

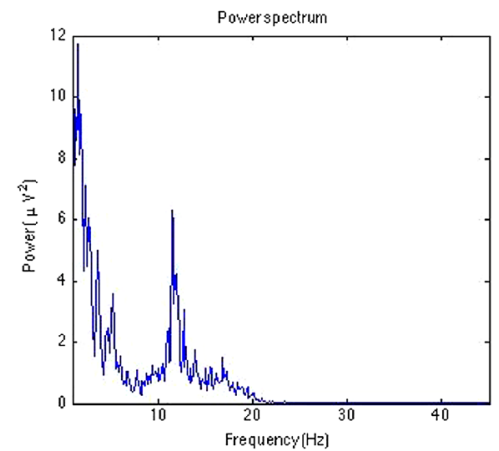

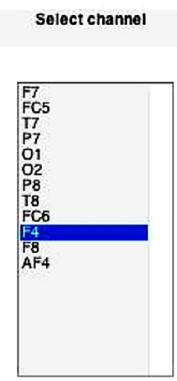

Fig. 4 Example of an individual channel from the right hemisphere of the brain. The colors on the top represent the activity of the specific brain area where the electrode was placed. The graph below witnesses

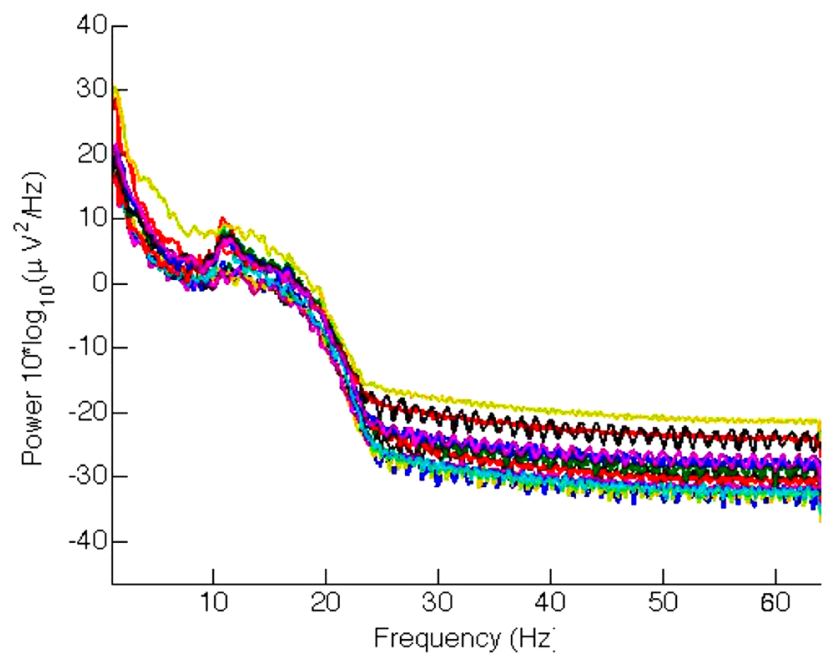

Fig. 5 Power spectrum plot for simple learning task in SL-group using NBT. We can witness that frequency peaks at $11-12 \mathrm{~Hz}$. The different colored lines show the respective channel (SL-groupsimple learning task NBT)

are characterized by alpha frequency on EEG with eyes open, while the deep relaxation coexists with both an increased internalized attention and an increased awareness of the environment. the power spectrum, and the dots in the right show the position of each electrode on the scalp (B-group_relaxation task)

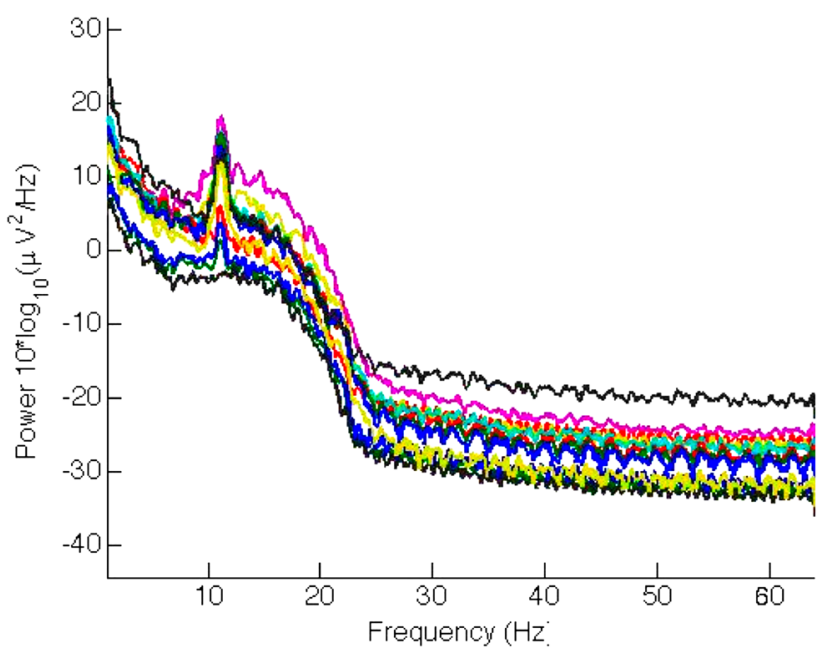

Fig. 6 Power spectrum plot for learning task in EL-group using NBT. We can witness the frequency peak at $11 \mathrm{~Hz}$. The colored lines indicate the different channels (EL-group-learning task NBT)

After having obtained the recorded EEG raw data from the Emotiv headset, we used EEGlab and NBT to remove possible artifacts and then to analyze and process the raw signals into a clean signal.

Our results clearly show that rhythmic audiovisual stimulation and relaxation in VR elicits in the subjects of 


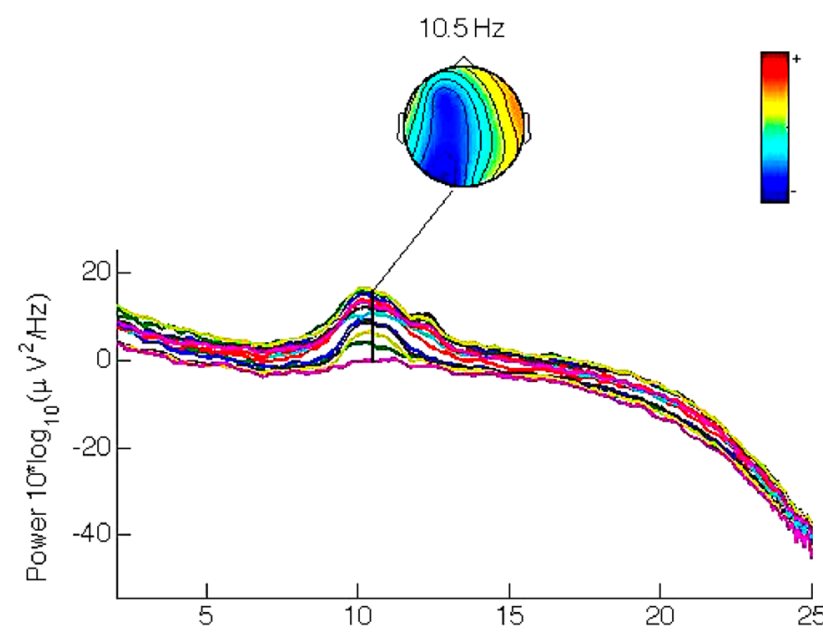

Fig. 7 Power spectrum plot for relaxation task in EFL-group using EEGlab. We witness that frequency peaks at $10 \mathrm{~Hz}$. The activity in the brain during this frequency is shown via the scalp map above the graph (EFL-group-relaxation task EEGlab)

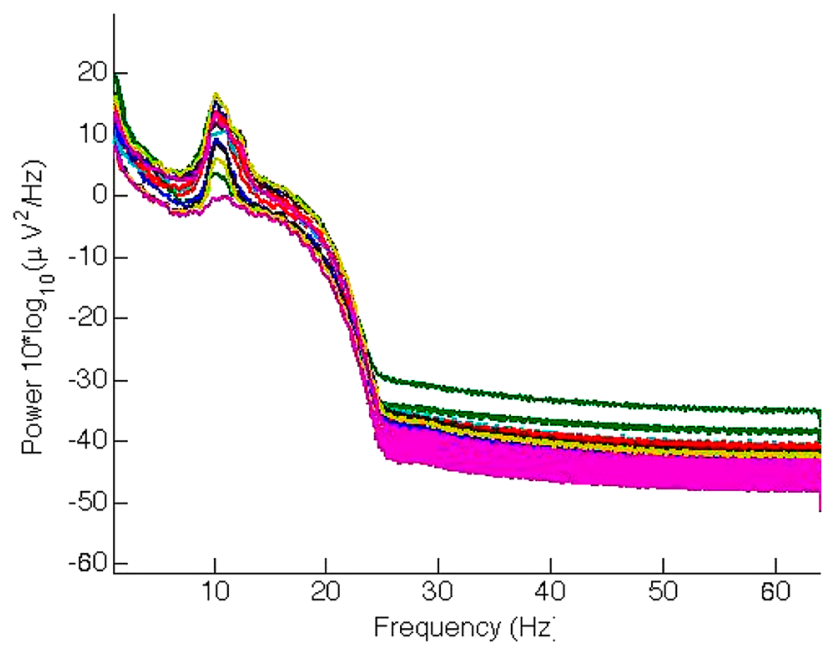

Fig. 8 Power spectrum plot for relaxation task in EFL-group using NBT. We can clearly witness the peak at $10 \mathrm{~Hz}$. (EFL-grouprelaxation task $N B T$ )

the $\mathrm{B}$, the EL and EFL-groups an increase in alpha brain waves $(8-12 \mathrm{~Hz})$. From our analysis, we also noticed in those subjects a significant presence of brainwaves in the delta range.

As rhythmic audio stimulation is what differentiates the SL- from the EL-group, and since the EL- and EFL-groups performed better in the labyrinth task than all the other groups (as we predicted in our theoretical model), we are prone to think that increase in alpha and delta brain waves is a key factor if we want to accelerate content presentation in VR for fast learning purpose.

Let's now discuss the experimental results presented in the Table 2 from the viewpoint of time related performance.
The first interesting result regards the effect-on the performance in the immediately subsequent labyrinth test-of rhythmic brain stimulation (entrainment) combined with the presentation of a relaxing environment in Oculus VR. We remind the reader that the B-group did not receive any form of learning. Previous researches seem to indicate that, in general, rhythmic brain stimulation alone can help learning and performance, if such performance involves aspects of creativity in a relaxed free-of-pressure artistic environment, speed of execution is not a relevant factor and tasks are not cognitively complex. If that can be true under certain conditions, our experiment clearly showed that rhythmic brain stimulation immediately before a cognitive complex orientation performance, where time is a critical factor, is not the best choice. In fact three of the subjects in the B-group could not complete the labyrinth task under the time limit of 6 min, while the C-group could in less than $4 \mathrm{~min}$.

The above findings can be related to the nature of our experiment. Finding the halfway landmark and especially passing through the second part of the labyrinth, without previous knowledge of the passage from entrance to exit, were stressful situations eliciting the fight-flight response requiring hyper vigilance and increased sympathetic tone as well as increased muscle tone, according to the bodily changes described by Cannon [5] and Selye [36]. Deep relaxation, that is, ataraxia or the absence of anxiety [27], naturally decreases performance in the above tasks, because this condition is the opposite of stress.

As shown in Fig. 2, the time for all the subjects of the B group to find the halfway landmark was almost 5 times more than the control group, and 11 times more than the best performing group, the Entrainment Fast learning one, where rhythmic brain stimulation was also combined with a learning session at high speed.

Those results are too significant in size and consistency to be just a coincidence: They seem to clearly indicate that rhythmic brain stimulation at alpha frequency $(10 \mathrm{~Hz})$ and relaxation in VR is highly counterproductive if immediately after that the subject has to perform a complex operational task (especially when it involves real or imaginary motor movements)—where completion time is a significant factor creating stress.

Concerning SL- and EL-groups, we observe an interesting result. In both groups, training happened at normal speed. The only difference in experimental conditions is the presence of relaxation, in EL-group, in Oculus VR plus audio rhythmic brain stimulation, which are not present as conditions in the SL-group. Obviously both groups performed better than the C-group and the B-group, because the learning process of the subject, watching passively a first-person camera flying through the labyrinth from the entrance directly to the exit, had a significant positive 


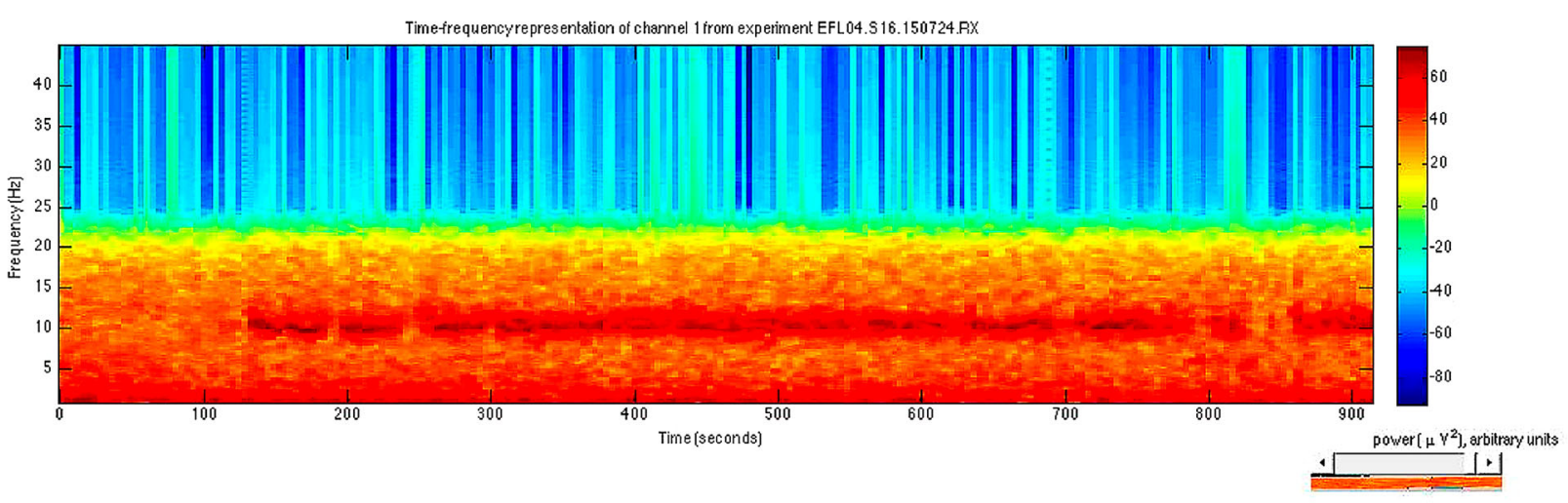

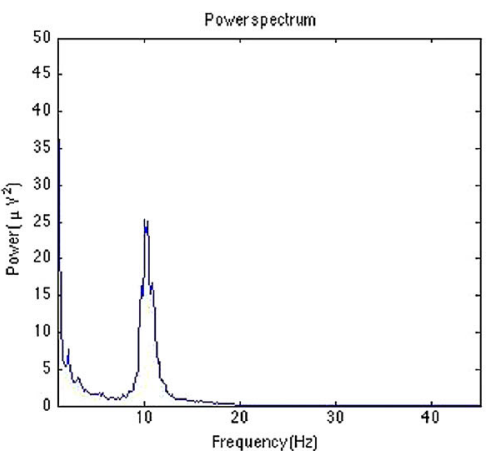

Fig. 9 Example of an individual channel from the right hemisphere of the brain in EFL-group using NBT. The colors on the top represent the activity of the specific brain area where the electrode was placed. The more intense the red color, the more intense the activity. The

effect on performance by reducing cognitive anxiety [18]. But among them, for SL- and EL-groups no significant difference was recorded in time to halfway landmark, which is the actual measure of effectiveness of learning procedure. EL-group, however, exhibited significantly lower time to pass through the stressful second part of the labyrinth, suggesting that the knowledge of how to pass through the labyrinth from the entrance directly to the exit (reducing cognitive anxiety) coupled with the induction of alpha rhythm (reducing arousal) increased the performance under stress [18].

So, as expected, it appears that relaxation/lowering brainwave frequency toward the alpha range and learning session at normal speed do not improve significantly the effectiveness of the learning process. If we consider on the other hand the EFL-group, which differs from the ELgroup only in the speed of content presentation in the learning phase, the results are quite different. In fact, the EFL-group performed to the halfway mark in average far better than the EL-group and SL-group, which even on already very fast performance of EL is a very significant improvement. When we compare the results of the SLgroup with those of the EL- and EFL-group, the graph below witnesses the power spectrum, and the dots in the right show the position of each electrode on the scalp (EFL-grouprelaxation task $N B T$ )

experimental findings confirm our research hypothesis that rhythmic brain stimulation and relaxation before a training session at an accelerated speed are a winning combination to produce the best result in direct learning and human enhancement.

Indirect learning of certain ability is the process where we learn how to develop such ability without undergoing any form of direct learning related specifically to that ability. In contrast direct learning is any kind of structured learning specifically related to that specific ability. A very interesting result has to do with the effect on indirect learning of our combined audio rhythmic brain stimulation and fast content presentation in VR. From our experimental results, it seems that not only such combination is effective for direct learning (as measured by time to landmark in the labyrinth test), but also very effective in indirect learning, and specifically on the ability we previously called: "precision and recovery." In the C-group, the difference between the halfway mark time and the exit time (the Delta) is around $191 \mathrm{~s}$ and that is our baseline. In the B-group, the rhythmic brain stimulation and the relaxation in VR seem to have completely compromised that "precision and recovery" ability naturally present in the 
Table 3 Summary of statistics for data variables T1 and TOT

\begin{tabular}{llllll}
\hline Group code & \multicolumn{1}{l}{ T1 } & & \multicolumn{2}{l}{ TOT } \\
\cline { 2 - 3 } & Average & Standard deviation & & Average & Standard deviation \\
\hline C & 35.5 & 4.79583 & 226.75 & 4.71699 \\
B & 133.25 & 4.64579 & & - & - \\
SL & 25.25 & 1.70783 & 102.0 & 1.82574 \\
EL & 23.5 & 2.38048 & 69.3333 & 2.51661 \\
EFL & 15.25 & 1.5 & 74.75 & 2.06155 \\
\hline
\end{tabular}

C-group: All the B-group subjects could find the halfway mark (but it took them much more than the C-group), but got lost in the second half of the labyrinth, where the only simple thing to do was to follow the only way till the exit. In the SL-group, even though the orientation learning had nothing to do with "precision and recovery," that ability was indirectly and significantly improved. A better result was in the EL-group, where learning at normal speed was combined with rhythmic brain stimulation and the relaxation in VR. In EL-group, the delta was more than 4 times less than the control group, and more than 1.5 time that of the SL-group. So, it seems that-in this case-it is actually the combination of learning and rhythmic brain stimulation and relaxation in VR that indirectly but significantly improved the ability of a subject to perform precise movements in the labyrinth and effectively recovering from accidental mistakes. The EFL-group performed slightly worse than the EL-group only because a single subject in the EFL-group performed terribly in the second half of the labyrinth. If we remove that subject from the EFL-group, average time to exit of that group decreases to $57.6 \mathrm{~s}$ (which is an improvement respect to the exit time of EL-Group) and the delta of EFL-group become about $42 \mathrm{~s}$, even better than the EL-group. So, also for indirect learning the EFL-group performed better than the other groups.

Finally, there is a possibility that the EFL-group performed better than the other groups, because the "peripheral route" according to the [31] communication model was activated in this group. Thus, when the learning video at fast speed was presented, information was passed directly to the right hemisphere, which is involved in spatial perception and navigation through the labyrinth [40] without the subject's careful and thoughtful consideration of the information presented.

Let T1 be "time to halfway mark in the maze" and TOT $=$ total time to exit. Regarding TOT, the B group was not part of this statistical analysis as 3 B-subjects out of 4 did not complete the labyrinth task within the 6-min time limit. One subject also of the EL-group did not complete the labyrinth task within the 6-minute time limit. For this reason, and because the n.a. values were dealt with as "missing values," the B group regarding TOT was not part of the ANOVA calculation. Results of the statistical analysis for the data variables T1 and TOT are illustrated in Table 3.

There is a statistically significant difference between the mean $\mathrm{T} 1$ from one level of code to another $(\mathrm{p}<0.01)$. Four homogeneous groups are identified (1. EFL, 2. EL together with SL, 3. C and 4. B, respectively). Here EFL constitutes by itself a homogeneous group characterized by the shortest time interval, it is followed by EL together with SL and then the final two (C and B).

Finally, there is a statistically significant difference between the mean TOT from one level of code to another $(\mathrm{p}<0.01)$. Three homogeneous groups are identified (1. EL together with EFL, 2. SL and 3. C, respectively). Here EL together with EFL constitute together a homogeneous group characterized by the shortest time interval, followed by SL- and finally the C-group (the B-group does not participate here as explained before) (Table 4).

In conclusion, the best performing group in the labyrinth test was the EFL-group, considering both the direct effectiveness of learning in exiting the maze, and the indirect effectiveness of learning in increasing the ability to perform precise movements in the labyrinth and effectively recovering from accidental mistakes while doing so. This fact was scientifically proven by the statistics as illustrated in the previous sections.

Table 4 Significant differences between groups according to ANOVA test using multiple range tests for $\mathrm{T} 1$

\begin{tabular}{lccc}
\hline Groups contrast & Sig. & Difference & \pm Limits \\
\hline C-B & $*$ & -97.75 & 5.0176 \\
C-SL & $*$ & 10.25 & 5.0176 \\
C-EL & $*$ & 12.0 & 5.0176 \\
C-EFL & $*$ & 20.25 & 5.0176 \\
B-SL & $*$ & 108.0 & 5.0176 \\
B-EF & $*$ & 109.75 & 5.0176 \\
B-EFL & $*$ & 118.0 & 5.0176 \\
SL-EF & & 1.75 & 5.0176 \\
SL-EFL & $*$ & 10.0 & 5.0176 \\
EF-EFL & $*$ & 8.25 & 5.0176 \\
\hline
\end{tabular}

* denotes a statistically significant difference at the $95 \%$ confidence level 
Considering that the learning time for the subject in the EFL-group was nearly half of the learning time spent by the other learning group (SL and EL-group), this result seems to indicate that audio rhythmic stimulation at alpha frequency combined with fast content presentation in VR is the most effective tool for learning and human enhancement, at least in the context of our experiment. Further research and experiments are necessary to determine upper limits of the effectiveness of this procedure and for which area of human enhancement this procedure is applicable. If those findings could be vastly applied, and learning time could be reduced to half for a large number of applications, the impact on education and human evolution could be measurably substantial.

The statistical significance of the research might be arguable, as the experimental group was limited to only 25 subjects, the experimental groups many (5) and the experimental conditions were complex (a complex VR environment with many variables). Nevertheless, our main objective is the practical realization of a "proof of concept." The purpose of this preliminary prototype and research is to prove a concept that the theoretical idea of fast learning in VR is-under certain conditions-indeed feasible and has important practical potential.

\section{Discussion}

The experimental findings in our research indicate that neuro- and biofeedback systems for Real-time, Autonomous Cognitive State Detection, Manipulation and Augmentation, audiovisual rhythmic brain stimulation can be combined with increased speed in content presentation and retention in latest virtual reality (VR) immersive headmounted displays. This approach seems to be the most appropriate strategy to improve particular learning and problem-solving abilities related to the presented experimental conditions. This work also illustrates that-under certain conditions-learning time can be drastically reduced at least to the half by utilizing audiovisual rhythmic brain stimulation and immersive VR, while at the same time increasing task performance. Further research and experiments are needed to establish into what extent and in which areas of human performance our novel architecture for augmented cognition can be effectively used to improve general human learning and problem-solving abilities.

Areas of future-related research are:

- Integrating more complex neuro- and biofeedback measurement for effective Real-time, Autonomous Cognitive State Detection, Manipulation and Augmentation to increase speed of learning and performance enhancement;
- Experimentally and theoretically establish the limits to which it is possible — by utilizing our novel architecture for augmented cognition-to reduce learning time while at the same time increasing learning effectiveness and problem-solving abilities in different contexts;

- Define maximum possible variable speed of content presentation in VR so that the subject's brain can still effectively process them;

- Define the best complex cognitive state, related to and measurable by integrated neuro- and biomarkers to improve learning, problem-solving abilities and performance.

- Investigating and developing fast learning tools for a wide variety of skill and bodies of knowledge that uses content presentation in VR adapting real-time to neuroand biological condition of the subject

Acknowledgements The research leading to these results has received funding from the EU FET Proactive grant TIMESTORMMind and Time: Investigation of the Temporal Traits of HumanMachine Convergence, funded under H2020-EU.1.2.2.-FET Proactive, with reference 641100

\section{References}

1. Adrian E, Matthews B (1934) The Berger rhythm: potential changes from the occipital lobes in man. Brain 57:355-384

2. Beller M, Or E (1998) The crossroads between lifelong learning and information technology a challenge facing leading universities. J Comput-Med Commun 4(2). doi:10.1111/j.1083-6101. 1998.tb00094.x

3. Bilinskis I (2007) Digital alias-free signal processing, pp 14-17 and 71-76. ISBN 978-0-470-02738-7

4. Bostrom N (2002) Existential risks: analyzing human extinction scenarios and related hazards. J Evol Technol 9(1):1-30

5. Cannon WB (1915) Bodily changes in pain, hunger, fear and rage: an account of recent researches into the function of emotional excitement. Appleton-Century-Crofts, New York

6. Casciaro F, Laterza V, Conte S, Pieralice M, Federici A, Todarello A, Orsucci F, Conte E (2013) Alpha-rhythm stimulation using brain entrainment enhances heart rate variability in subjects with reduced HRV. World J Neurosci 3(4):213-220

7. Chaitran G, Petersen M, Lazarte J (1959) Response to clicks from the human brain: Some depth electrographic observations. Electroencephalogr Clin Neurophysiol 12:479-489

8. Crow FC (1987) The aliasing problem in computer-generated shaded images. Mag Commun ACM 20:799-805

9. Dempsey E, Morison R (1942) The interaction of certain spontaneous and induced cortical potentials. Am J Physiol 135:301-307

10. Delorme A, Makeig S (2004) EEGLAB: an open source toolbox for analysis of single trial EEG dynamics including independent component analysis. J Neurosci Methods 134(1):9-21

11. Dennet CD, Kinsbourne D (1992) Time and the observer: the where and when of consciousness in the brain. Behav Brain Sci 15(2):183-201

12. Duvinage M, Castermans T, Petieau M, Hoellinger T, Cheron G, Dutoit T (2013) Performance of the Emotiv Epoc headset for 
P300-based applications. BioMed Eng OnLine 12:56. doi:10. $1186 / 1475-925 X-12-56$

13. Goldman G, Segal J, Segalis M (1938) L'action d'une excitation inermittente sur le rythme de Berger (The effects of intermittent excitation on the Berger rhythms), vol 127. C.R Societe de Biologie, Paris, pp 1217-1220

14. Gruzelier JH (2008) A theory of alpha/theta neurofeedback, creative performance enhancement, long distance functional connectivity and psychological. Cogn Process. doi:10.1007/ s10339-008-0248-5

15. Gruzelier J, Inoue A, Smart R, Steed A, Steffert T (2010) Acting performance and flow state enhanced with sensory-motor rhythm neurofeedback comparing ecologically valid immersive VR and training screen scenarios. Neurosci Lett 480(2):112-116

16. Gruzelier JH, Foks M, Steffert T, Chen MJL, Ros T (2014) Beneficial outcome from EEG-neurofeedback on creative music performance, attention and well-being in school children. Biol Psychol 95:86-95

17. Gruzelier JH, Thompson T, Redding E, Brandt R, Steffert T (2013) Application of Alpha/Theta neurofeedback and heart rate variability training to young contemporary dancers (State anxiety and creativity). Int J Psychophysiol 93(1):105-111

18. Hardy L, Parfitt G (1991) A catastrophe model of anxiety and performance. Br J Psychol 82(2):163-178

19. Hilbert M, López, P (2011) The world's technological capacity to store, communicate, and compute information. Science 332(6025):60-65. doi:10.1126/science.1200970. PMID 21310967

20. Huang TL, Charyton C (2008) A comprehensive review of the psychological effects of brainwave entrainment. Altern Ther Health Med 14(5):38-49

21. Jung R (1973) Das Elektroencephalogram und seine klinische Anwendung, (The electroencephalogram ans its clinical application. Nervenarzt 12:569-591

22. Kasamatsu KH, Hirai T (1996) An electroencephalographic study on the zen meditation (Zazen). Folia Psychiatrica et Neurologica Japonica 20(4):315-336

23. Kateros S, Georgiou S, Papaefthymiou M, Papagiannakis G, Tsioumas M (2015) A Comparison of gamified, immersive VR curation methods for enhanced presence and human-computer interaction in digital humanities. Int J Heritage Digit Era 4(2):221-234. doi:10.1260/2047-4970.4.2.221

24. Krugman HE, Hartley EL (1970) Passive learning from television. Oxford J 34:184-190

25. Lagopoulos J, Xu J, Rasmussen I, Vik A, Malhi GS, Eliassen CF, Arntsen IE, Saether JG, Hollup S, Holen A, Davanger S, Ellingsen $\varnothing$ (2009) Increased theta and alpha EEG activity during nondirective meditation. J Altern Complement Med 15(11):1187-1192

26. Mathewson KE, Basak C, Maclin EL, Low KA, Boot WR, Kramer AF, Fabiani M, Gratton G (2012) Different slopes for different folks: alpha and Beta EEG power predict subsequent video game learning rate and secondary task improvement. Psychophysiology 49:1558-1570
27. Nestoros JN (1984) GABAergic mechanisms and anxiety: an overview and a new neurophysiological model. Can J Psychiatry 28(6):520-529

28. Oster G (1973) Auditory beats in the brain. Sci Am 4:94-102

29. Papaefthymiou M, Feng A, Shapiro A, Papagiannakis G (2015) A fast and robust pipeline for populating mobile AR scenes with gamified virtual characters. In: ACM SIGGRAPH-ASIA 2015, symposium on mobile graphics and interactive applications, Kobe. ACM Press

30. Papagiannakis G, Papanikolaou P, Greassidou E, Trahanias PE (2014) glGA: an OpenGL geometric application framework for a modern, shader-based computer graphics curriculum, eurographics. Edu Pap 2014:9-16

31. Petty RE, Cacioppo JT (1986) Communication and persuasion: central and peripheral routes to attitude change. New York, NY: Springer

32. Schier MA (2000) Changes in EEG alpha power during simulated driving: a demonstration. Int J Psychophysiol 37(2):155-162

33. Schmorrow D (2014) Augmented cognition: achieving human computer symbiosis with 21 st century human factors tools, Tutorial notes. HCI International, Heraklion

34. Schmorrow D, Kruse A (2002) DARPA's Augmented Cognition Program-tomorrow's human computer interaction from vision to reality: building cognitively aware computational systems. Hum Factors Power Plants 1:4. doi:10.1109/HFPP.2002.1042859

35. Schmorrow D, John StM, Kobus DA, Morrison JG (2004) Overview of the DARPA augmented cognition technical integration experiment. Int J Hum-Comput Interact 17(2):131-149

36. Selye H (1955) Stress and disease. Science 22(3171):625-631

37. Siever D (2003) Audio-visual entrainment: history and physiological mechanisms. Biofeedback 31:21-27

38. Stanney KM, Schmorrow DD, Johnston M, Fuchs S, Jones D, Hale KS, Ahmad A, Young P (2009) Rev Hum Factors Ergon 5(1):195-224

39. Toman J (1941) Flicker potentials and the alpha rhythm in man. J Neurophysiol 4:51-61

40. Toga AW, Thompson PM (2003) Mapping brain asymmetry. Nat Rev Neurosci 4:37-48

41. The Role of Science and Technology in GEOSS (prepared by the GEO Science and Technology Committee)

42. Thompson T, Steffert T, Ros T, Leach J, Gruzelier J (2008) EEG applications for sport and performance. Methods 45(4):279-288

43. Walter GW (1956) Colour illusions and aberrations during stimulation by flickering light. Nature 177:710

44. Yudkowsky E, Bostrom N, Cirkovic M (2014) Artificial intelligence as a positive and negative factor in global risk. Global Catastrophic Risks. Oxford University Press, p 303, Bibcode:2008gcr..book..303Y, ISBN 978-0-19-857050-9

45. Zhuang T, Zhao H, Tang Z (2009) An Analysis of improving memory performance based on EEG alpha and theta oscillations. Comput Inf Sci 2(1):108-114 